\title{
Design of Microwave Pulse Compressors Using Small Form-Factor Waveguide Cavities
}

\author{
Z. C. Ioannidis, S. P. Savaidis, S. A. Mitilineos, S. Livieratos and N. A. Stathopoulos
}

\begin{abstract}
A microwave pulse compressor consisting of an iris, a straight waveguide section and a $T$-junction can be used in order to convert a long pulse with lower power to a shorter pulse with higher power. Provided that the system is designed precisely, a significant compression gain can be achieved. The duration of the compressed pulse is proportional to the cavity length, thus imposing a constraint to the development of mobile systems. In this work we present the circuit/transmission-line based design methodology that we follow in order to fold the cavity (while retaining the same electrical length) by using $T$ junction turns. As an example, we design a $1.3 \mathrm{GHz}$ folded compressor and we compare its performance to the corresponding one of a straight-cavity compressor. 3D full-wave simulations with CST Studio Suite and experimental measurements with the developed compressor confirm the accuracy of the proposed design methodology.
\end{abstract}

Index Terms-High-power microwaves, microwave pulse compression (MPC), T-junction, waveguide cavities.

\section{INTRODUCTION}

$\mathrm{M}$ ICROWAVE pulse compression is a rather simple and cost-efficient method in order to convert a long pulse of low power to a shorter one with higher power. In this way, it is possible to achieve very high power, even in the GW range, when this is not directly possible by a microwave oscillator or amplifier. Despite that this technique is well-known and used for large particle accelerators, such as SLED-II [1], it can be extremely useful to any other field application where a relatively wideband signal of high amplitude can be beneficial, e.g. long-range high resolution radar [2] or RF directed energy systems [3].

Usually, the Microwave Pulse Compressors (MPC) are divided to active and passive, depending on the approach used in order to store and release the energy [2]. Passive MPCs use a dispersive circuit such as a waveguide section and require a phase modulated input pulse. In general, the dispersive properties of simple waveguides are notable only near cutoff and for this reason more complex slow-wave structures are

Manuscript received March 01, 2020; revised April 24, 2020; accepted April 28, 2020 (Corresponding author: Zisis Ioannidis)

Z. C. Ioannidis is with the Institute for Pulsed Power and Microwave Technology (IHM) at the Karlsruhe Institute of Technology (KIT), Karlsruhe, 76131, Germany (email: zisis.ioannidis@kit.edu).

S. P. Savaidis, S. A. Mitilineos and N. A. Stathopoulos are with the WaveComm Lab at the University of West Attica, Aigaleo, 12244, Greece.

S. Livieratos is with the Department of Electrical and Electronic Engineering at the School of Pedagogical and Technological Education, Iraklion, 14121, Greece. usually employed [4] to work at higher frequencies.

The operation of an active MPC is somewhat simpler and does not rely on the dispersion of the medium. In particular, a relatively low-power source is used in order to inject power into a high quality-factor cavity and to create a standing wave. The cavities that are, usually, used for the storage of the energy are straight waveguides that are connected on their open ends with highly-reflective components such as irises and shorted T-junctions. As soon as the cavity is loaded with energy, an active element such as a plasma switch [5], [6] is activated and the energy is released.

Provided that an active MPC is precisely designed and uses a fast enough switch, it has significant gain with fast rise-time and small precursor signals [7]. The duration of the output pulse is almost equal to the propagation round-trip time in the cavity [8]. Due to this fact, longer pulses would need inconveniently long cavities. For example, $10 \mathrm{~ns}$ pulses at $1.3 \mathrm{GHz}$ roughly need a $1 \mathrm{~m}$ long WR-650 waveguide cavity [8], [9]. This length explodes to multiple meters, when tenths of ns are necessary. Note that the MPC output pulse duration depends both on the group velocity and the physical length. Thus, even the migration to higher frequencies would not overcome the aforementioned limitation [8], [9].

Recently, an interesting idea was proposed in order to reduce the physical size of an active MPC, while retaining the electrical length of the cavity. In particular, in [10]-[13] it is proposed that by using additional passive microwave components, such as waveguide turns, it is possible to fold the resonant cavity to a meander shape and reduce the size of the system. The dimensions of the waveguide turns have to be precisely selected in order to maximize the storage capabilities of the complex cavity. This scheme was used for an MPC working at $2.8 \mathrm{GHz}$ made of cylindrical waveguides and Tjunctions [10], [11]. However, due to the intuitive design there is no guarantee that the overall gain that was achieved is the maximum possible. Such a gain optimization becomes a critical issue, since the increase of the MPC electrical length inherently suppresses the cavity gain [8], [9]. A simple yet effective approach to optimize the dimensions of a waveguide based MPC and maximize the cavity gain has been presented in [9], [14] and [15].

In this work we employ the transmission-line/circuit approach to accurately design Folded Microwave Pulse Compressors (FMPC) consisting of multiple rectangular waveguide-cavities. In Section II, we study the combination of two H-plane T-junctions in order to optimize the properties of 
the corresponding waveguide turn. Then, in Section III, such a turn is introduced to the equivalent circuit of an FMPC, and the compressor dimensions that maximize the cavity gain are calculated. In Section IV, a $1.3 \mathrm{GHz}$ example design is presented and verified by 3D full-wave simulation. In Section $\mathrm{V}$, the proposed FMPC system is developed and tested experimentally. Our conclusions are summarized in Section VI.

\section{DESIGN OF MATChED H-Plane WAVEGUIDE TURNS}

It is possible to create rectangular waveguide-turns by connecting $\mathrm{H}$-plane $\mathrm{T}$-junctions. In order to minimize (maximize) the reflection coefficient $\mathrm{S}_{11}$ (transmission coefficient $S_{21}$ ) of the resulting two-port network, it is important to properly select the lengths of the T-junction arms. An efficient method is to calculate the scattering matrix of the turn by using the single T-junction equivalent circuit [16]. This approach is adequately accurate and much faster than full-wave simulation [9], [14].

Fig. 1 presents two H-plane T-junctions, connected at their auxiliary (bridge) arms, and short-circuited at one of their primary arms. The total bridge-arm length is equal to $w$ and the shorted-arm length is equal to $r$ (same for both sides). Both $w$ and $r$ parameters are normalized to the mode $\mathrm{TE}_{10}$ guided wavelength $\lambda_{\mathrm{g}}$. Fig. 2 presents the equivalent circuit of the above described T-junction turn. The core part of the Tjunction circuit, which is located inside the grey rectangles in Fig. 2, is considered to be lossless. On the contrary, the waveguide arms are represented as transmission lines with characteristic impedance $\mathrm{Z}_{0}$, attenuation factor $\alpha$ and propagation factor $\beta$, i.e. with propagation constant $\gamma=\alpha+\mathrm{j} \beta$. According to Fig. 2 the reflection coefficient $S_{11}$ is calculated as follows:

$$
S_{11}=\left(Z_{A A^{\prime}}-Z_{0}\right) /\left(Z_{A A^{\prime}}+Z_{0}\right),
$$

with $Z_{\mathrm{AA}}$, calculated from the equations:

$$
\begin{gathered}
Z_{A A^{\prime}}=Z_{B A^{\prime}}+j X_{a} \\
Z_{B A^{\prime}}=\left(Z_{0} \tanh \left(\gamma \cdot r \cdot \lambda_{g}\right)+j X_{a}\right) \|\left(Z_{C A^{\prime}}+j X_{b}\right) \\
Z_{C A^{\prime}}=\left(Z_{D A^{\prime}}+j X_{d}\right) \| j X_{c} \\
Z_{D A^{\prime}}=Z_{0} \frac{Z_{E G^{\prime}}+Z_{0} \tanh \left(\gamma \cdot w \cdot \lambda_{g}\right)}{Z_{0}+Z_{E G^{\prime}} \tanh \left(\gamma \cdot w \cdot \lambda_{g}\right)} \\
Z_{E G^{\prime}}=\left(Z_{F G^{\prime}} \| j X_{c}\right)+j X_{d} \\
Z_{F G^{\prime}}=j X_{b}+Z_{G G^{\prime}} \\
Z_{G G^{\prime}}=\left(Z_{0} \tanh \left(\gamma \cdot r \cdot \lambda_{g}\right)+j X_{a}\right) \|\left(Z_{0}+j X_{a}\right)
\end{gathered}
$$

Assuming that the input and the forward voltages of the Tjunction are $V_{i n}$ and $V_{i n}^{+}$, respectively, the transmission coefficient $S_{21}$ is calculated by following the voltage dividers along the equivalent circuit (Fig. 2). In particular:

$$
S_{21}=V_{Z_{I G^{\prime}}} / V_{\text {in }}^{+},
$$

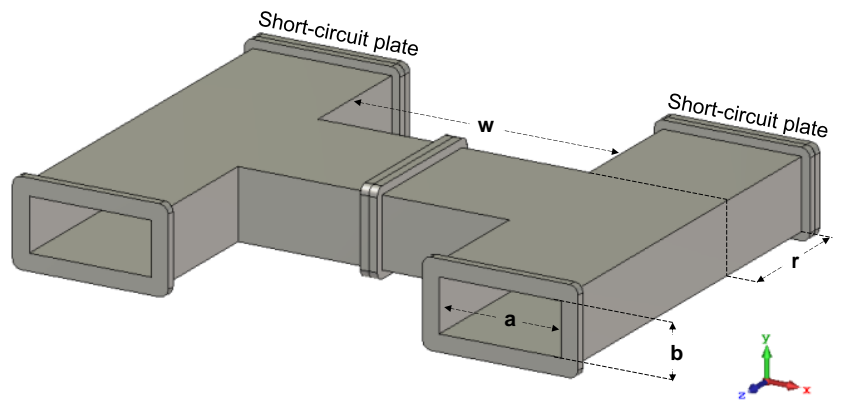

Fig. 1. H-plane T-junctions connected at their auxiliary arms in order to form a waveguide turn. The primary output arms of both T-junctions are shorted.

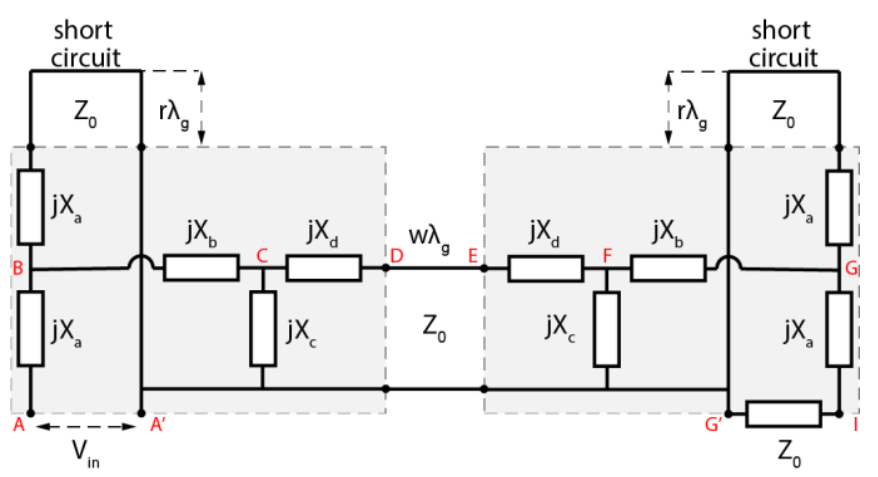

Fig. 2. Equivalent circuit of the connected H-plane T-junctions of Fig. 1.

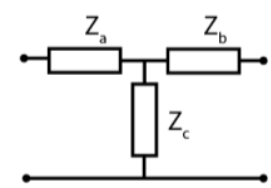

Fig. 3. T-network representation of the equivalent circuit presented in Fig. 2.

where

$$
\begin{aligned}
& V_{Z_{I G^{\prime}}}=V_{Z_{G G^{\prime}}} \cdot Z_{0} /\left(Z_{0}+j X_{a}\right) \\
& V_{Z_{G G^{\prime}}}=V_{Z_{F G^{\prime}}} \cdot Z_{G G^{\prime}} / Z_{F G^{\prime}} \\
& V_{Z_{F G^{\prime}}}=V_{Z_{E G^{\prime}}} \cdot\left(Z_{F G^{\prime}} \| j X_{c}\right) / Z_{E G^{\prime}} \\
& V_{Z_{E G^{\prime}}}=V_{Z_{D A^{\prime}}} /\left(\cosh \left(\gamma \cdot w \cdot \lambda_{g}\right)+Z_{0} \sinh \left(\gamma \cdot w \cdot \lambda_{g}\right) / Z_{E G^{\prime}}\right) \\
& V_{Z_{D A^{\prime}}}=V_{Z_{C A^{\prime}}} \cdot Z_{D A^{\prime}} /\left(Z_{D A^{\prime}}+j X_{d}\right) \\
& V_{Z_{C A^{\prime}}}=V_{Z_{B A^{\prime}}} \cdot Z_{C A^{\prime}} /\left(Z_{C A^{\prime}}+j X_{b}\right) \\
& V_{Z_{B A^{\prime}}}=V_{i n} \cdot Z_{B A^{\prime}} /\left(Z_{B A^{\prime}}+j X_{a}\right) \\
& V_{\text {in }}=\left(1+S_{11}\right) V_{\text {in }}^{+}
\end{aligned}
$$

The impedance matrix $\mathbf{Z}$ of the two-port network can be now calculated from the scattering matrix $\mathbf{S}$ as follows:

$$
\mathbf{Z}=(\mathbf{I}-\mathbf{S})^{-1} \cdot(\mathbf{I}+\mathbf{S})
$$

Based on equation (18), the combination of the T-junctions can be represented by the T-network of Fig. 3. The $Z_{\mathrm{a}}, Z_{\mathrm{b}}$ and $Z_{\mathrm{c}}$ impedances are given by:

$$
\begin{gathered}
Z_{a}=Z_{b}=Z_{11}-Z_{12} \\
Z_{c}=Z_{12},
\end{gathered}
$$




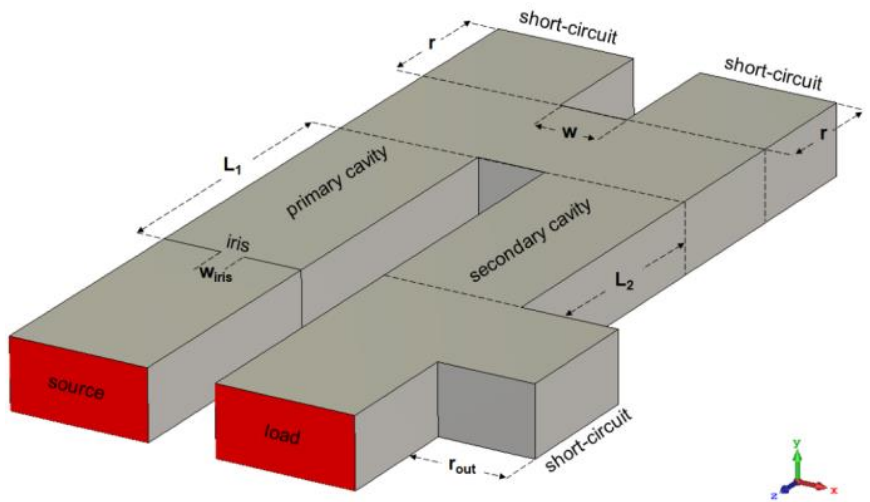

Fig. 4. Folded Microwave Pulse Compressor using an H-plane turn.

where $Z_{11}$ and $Z_{12}$ are the terms of the first line of the impedance matrix [17].

It is straightforward to do a similar analysis for two E-plane T-junctions connected at their auxiliary arms. In such case it is more convenient to work with the admittance matrix, since the equivalent circuit is usually represented with admittances. However, E-plane turns are less preferable for high-power applications (low electrical strength) and thus this alternative design will not be discussed further.

\section{FMPC SYSTEM UTILIZING AN H-PLANE TURN}

Fig. 4 presents a FMPC, which uses an H-plane waveguideturn. The energy storage cavity consists of a primary and a secondary part with lengths $L_{1}$ and $L_{2}$, respectively. Of course the total equivalent cavity length, the one that is related to the duration of the compressed pulse, is expected to be slightly larger, accounting for the additional length of the $\mathrm{T}$-junction turns. The leakage of the energy is controlled at the input of

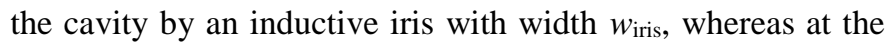
output of the cavity by a T-junction with shorted side-arm length $r_{\text {out }}$ (Fig. 4).

The corresponding circuit/transmission-line model is shown in Fig. 5. In this circuit, the waveguide turn has been replaced by its equivalent T-network as it is discussed in Section II. Assuming that the waveguide length $L_{2}$ is constant, it is possible to further simplify this circuit to the one presented in Fig. 6. Essentially, this circuit is identical to the one for the simple case of a straight-cavity MPC (SMPC) as it is presented in [9], [14]. In brief, [14] uses an iterative procedure to calculate the optimal values for the waveguide cavity length, the opening of the inductive iris and the length of the short circuit at the output port of the compressor. The same procedure can be followed here, provided that the load impedance of Eq. (2) in [14] is replaced by the corresponding load impedance $Z_{\mathrm{L}}^{\prime}$ calculated by Fig. 5. In particular:

$$
\begin{gathered}
Z_{L}^{\prime}=Z_{a}+Z_{c} \|\left(Z_{b}+Z_{i n, 2}\right) \\
Z_{i n, 2}=Z_{0} \frac{Z_{L}+Z_{0} \tanh \left(\gamma \cdot L_{2} \cdot \lambda_{g}\right)}{Z_{0}+Z_{L} \tanh \left(\gamma \cdot L_{2} \cdot \lambda_{g}\right)}
\end{gathered}
$$

where $\mathrm{Z}_{\mathrm{L}}$ is given by Eq. (2) in [14], i.e.:

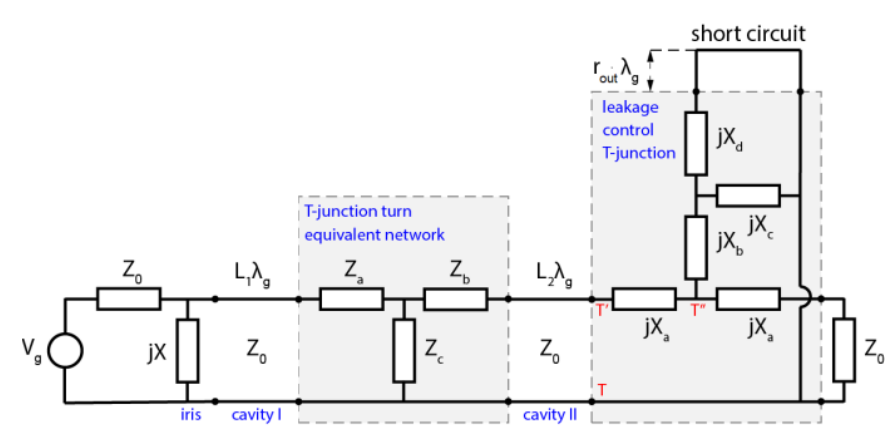

Fig. 5. Equivalent circuit of the folded compressor presented in Fig. 4.

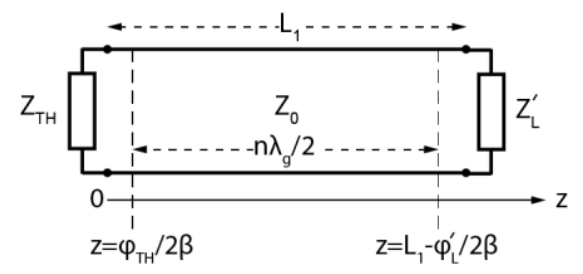

Fig. 6. Simplified folded cavity model as a transmission line terminated at both ends. $Z_{\mathrm{L}}^{\prime}$ is given by Eq. (21), whereas $\mathrm{Z}_{\mathrm{th}}$ is given by Eq. (1) in [14].

$$
Z_{L}=j X_{a}+\frac{Z_{S H}\left(Z_{0}+j X_{a}\right)}{Z_{0}+Z_{S H}+j X_{a}}
$$

and $Z_{S H}$ is the input impedance towards the shorted auxiliary arm of the turn and is given by Eq (3) in [14], i.e.:

$$
Z_{S H}=j X_{b}+\frac{j X_{c}\left(j X_{d}+Z_{S C}\right)}{j X_{c}+X_{d}+Z_{S C}}
$$

and the impedance $Z_{\mathrm{SC}}$, which controls the leakage of the compressor is given by:

$$
Z_{S C}=Z_{0} \tanh \left(\gamma \cdot r_{\text {out }}\right)
$$

similarly to Eq. (4) in [14].

The system of Eqs. (5) and (8) in [14] for the calculation of the optimal iris opening, cavity length and leakage-control short-circuit length, respectively, still holds. This is also true for the calculation of the cavity gain (Eq. (10) in [14]). The side cavity gain and the leakage have to be adapted in order to account for the additional part of the storage cavity (waveguide turn and second straight part). Based on the equivalent circuits in Fig. 5 and Fig. 6 the key features of the compression system are:

$$
\begin{gathered}
L_{1}=n \frac{\lambda_{g}}{2}+\frac{\varphi_{T H}+\varphi_{L}^{\prime}}{2 \beta} \\
X=Z_{0} \frac{\sqrt{1-\left|\rho_{L}^{\prime}\right|^{2} e^{-4 a L_{1}}}}{2\left|\rho_{L}^{\prime}\right| e^{-2 a L_{1}}},
\end{gathered}
$$

where $\beta$ is the phase constant $\varphi_{\mathrm{TH}}$ and $\varphi_{L}^{\prime}$ are the angles of the reflection coefficients of the terminating impedances $Z_{\mathrm{TH}}$ and 
$Z_{L}^{\prime}$, respectively. Considering that the standing wave along the primary cavity is given by:

$$
V(z)=\frac{V_{g}}{2} \frac{Z_{L}^{\prime} \cosh \left[\gamma \cdot\left(L_{1}-z\right)\right]+Z_{0} \sinh \left[\gamma \cdot\left(L_{1}-z\right)\right]}{Z_{L}^{\prime} \cosh \left(\gamma \cdot L_{1}\right)+Z_{0} \sinh \left(\gamma \cdot L_{1}\right)}
$$

the main cavity gain and the output leakage are equal to:

$$
\begin{gathered}
G=20 \log \left(\left|\frac{V\left(z=\varphi_{T H} / 2 \beta\right.}{V_{g} / 2}\right|\right) \\
L_{\text {out }}=20 \log \left(\left|\frac{V_{\text {out }}}{V_{g} / 2}\right|\right),
\end{gathered}
$$

where $\mathrm{V}_{\text {out }}$ is the voltage of the standing wave at the position of the output load, i.e.

$$
\begin{gathered}
V_{\text {out }}=\frac{V\left(L_{1}\right) \cdot Z_{0}}{Z_{0}+i X_{a}} \frac{Z_{S H} \|\left(Z_{0}+i X_{a}\right)}{Z_{S H} \|\left(Z_{0}+i X_{a}\right)+i X_{a}} \frac{Z_{i n, 2}}{Z_{b}+Z_{i n, 2}} . \\
\frac{Z_{c} \|\left(Z_{b}+Z_{i n, 2}\right)}{Z_{c} \|\left(Z_{b}+Z_{i n, 2}\right)+Z_{a}} \frac{Z_{L}}{Z_{L} \cosh \left(\gamma L_{2}\right)+Z_{0} \sinh \left(\gamma L_{2}\right)}
\end{gathered}
$$

Summarizing, we can calculate the optimal values of the key features of the FMPC by using equations (26) - (31). Despite that the methodology was presented for H-plane waveguide turns, any kind of turn can in principle be used, provided that it can be represented by a T-network such the one of Fig. 3.

\section{IV. $1.3 \mathrm{GHZ}$ FMPC DESIGN EXAMPLE}

The equivalent circuit/transmission-line model is used in order to optimize an FMPC design using WR-650 waveguides and an $\mathrm{H}$-plane turn. At $1.3 \mathrm{GHz}$ the single T-Junction equivalent circuit has terms $X_{\mathrm{a}} / Z_{0}=-4.96, \quad X_{\mathrm{b}} / Z_{0}=3.51$, $X_{\mathrm{c}} / Z_{0}=-0.68$ and $X_{\mathrm{d}} / Z_{0}=2.09$, which have been calculated from the graphical results in [16]. The waveguide attenuation factor is $\alpha=0.0013 \pm 0.005 \mathrm{~Np} / \mathrm{m}$, according to VNA measurements [18].

\section{A. Optimization of the H-Plane waveguide turn}

Fig. 7 presents the scattering matrix parameters $S_{11}$ and $S_{12}$, calculated using Eqs. (1) and (9), respectively, for different combinations of the bridge-arm length $w$ and the shorted-arm length $r$. For every $r$-value it is possible to find a $w$-value that ensures that the waveguide turn will be well matched. It is preferable to avoid, however, the region $0.15 \lambda_{\mathrm{g}}<r<0.30 \lambda_{\mathrm{g}}$, where the reflection coefficient strongly depends on $r$ and eventually on fabrication tolerances. Contrary, the ranges $0.0 \lambda_{\mathrm{g}}<r<0.15 \lambda_{\mathrm{g}}$ and $0.30 \lambda_{\mathrm{g}}<r<0.5 \lambda_{\mathrm{g}}$ are more appropriate and ensure that small fabrication inaccuracies of the $(r, w)$ combination will not deteriorate significantly the performance of the turn.

A similar discussion can be made for the appropriate ranges of the $w$-value. In practice, it is easier to control the $r$-value by
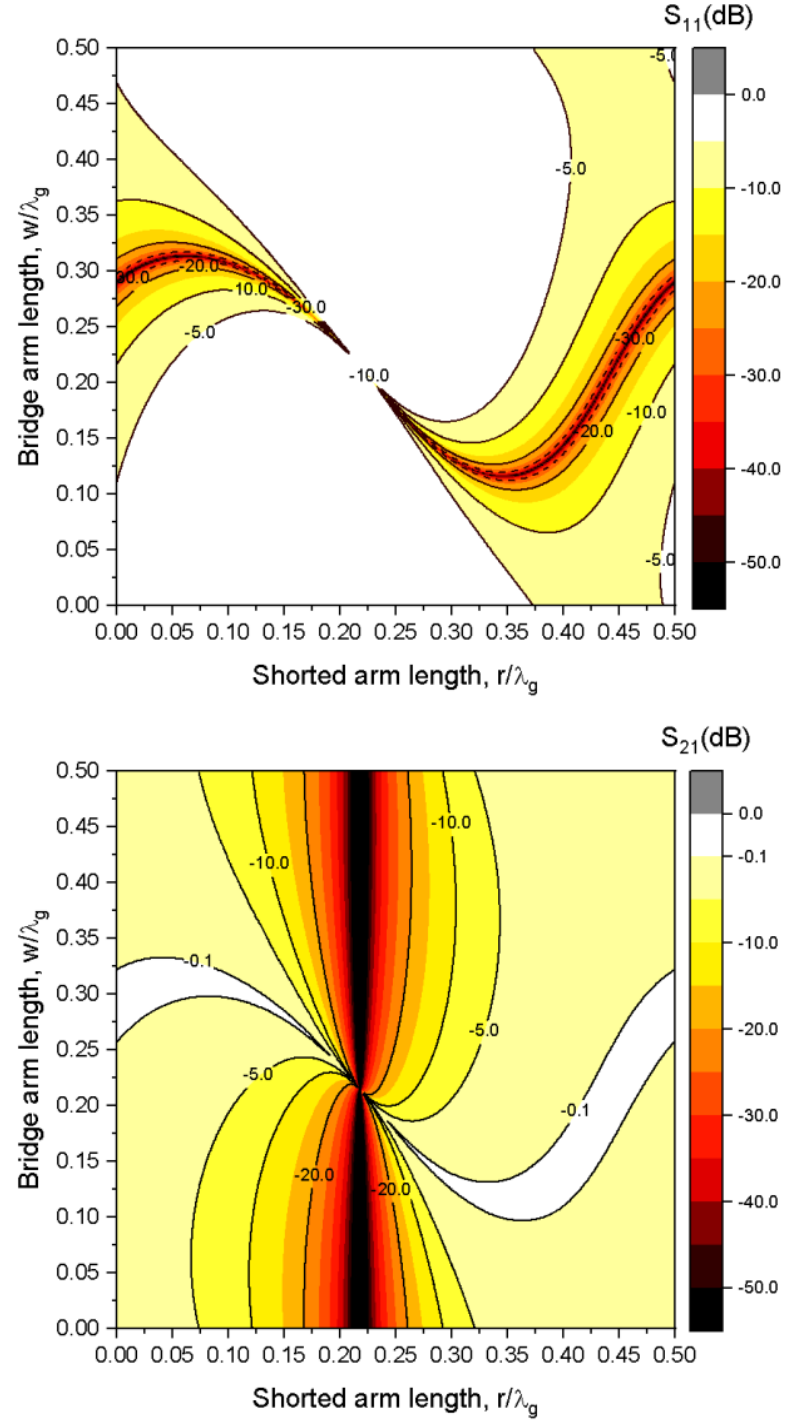

Fig. 7. S-matrix parameters for the two-port network presented in Fig. 1. The calculations correspond to WR-650 T-junctions operating at $1.3 \mathrm{GHz}$.

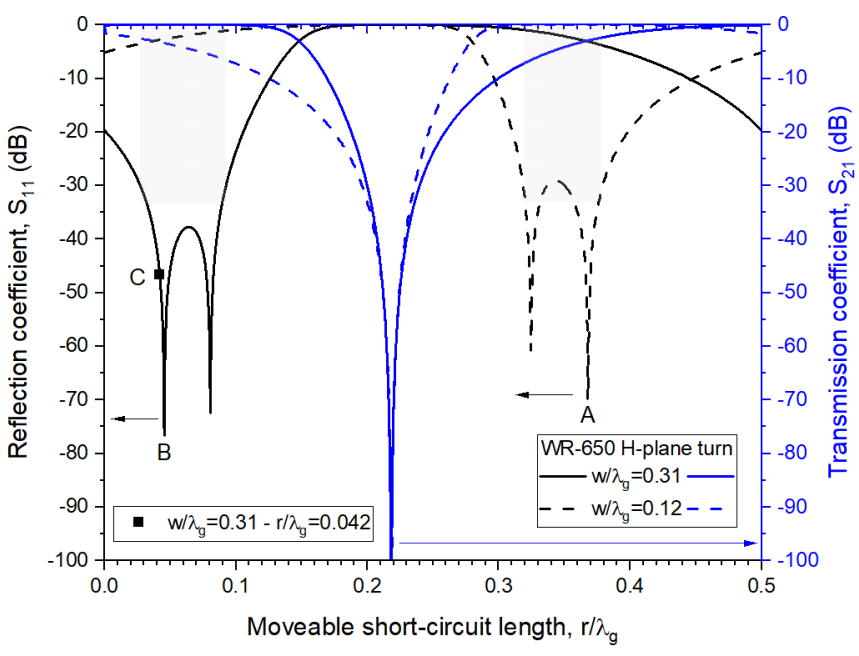

Fig. 8. Reflection and transmission coefficients for H-plane waveguide turns with bridge arm lengths $\mathrm{w}=0.31 \lambda_{\mathrm{g}}$ (solid curves) and $\mathrm{w}=0.12 \lambda_{\mathrm{g}}$ (dashed curves). The black square corresponds to the dimensions relevant to the experimental verification of the FMPC design. 
using a movable short-circuit than to change the $w$-value by using waveguide spacers. For this reason, it is better to consider the length $w$ of the bridge-arm as a design parameter and the short-circuit length $r$ as a product of optimization. For the example under discussion, there are two $w$-value areas that are preferable, namely $w \sim 0.12 \lambda_{\mathrm{g}}$ and $w \sim 0.31 \lambda_{\mathrm{g}}$. As shown in Fig. 7, both selections result to a reflection coefficient below $-30 \mathrm{~dB}$ and a transmission coefficient above $-0.1 \mathrm{~dB}$ in a $r$-value range wider than $0.05 \lambda_{\mathrm{g}}$, which corresponds to $16 \mathrm{~mm}$ at $1.3 \mathrm{GHz}$. The performance of the turn is expected to be relatively insensitive to manufacturing errors in this range.

Fig. 8 presents the scattering matrix parameters for both values of the two aforementioned bridge-arm lengths. The broadband behavior of the component, which is more evident in this two dimensional representation, is very beneficial for the design of the waveguide turn and supports the choice of using the $w$-value as a design parameter as the short circuit length can be easier tuned. The case of $w=0.12 \lambda_{\mathrm{g}}$ results to $r=0.368 \lambda_{\mathrm{g}}$ (point A), which can be easily set with a movable short, especially considering the reduced sensitivity of the reflection coefficient. Similarly, the case of $w=0.31 \lambda_{\mathrm{g}}$ results to $r=0.036 \lambda_{\mathrm{g}}$ (point $\mathrm{B}$ ).

For the design that we develop and study experimentally, we select $w=0.310 \lambda_{\mathrm{g}}$ and $r=0.542 \lambda_{\mathrm{g}}$. This choice is based on the dimensions of the T-junction that we have available at the laboratory and ensures that the minimum number of waveguide spacers is used. It should be noted that we have to select $r=0.542 \lambda_{\mathrm{g}}$ (instead of $0.042 \lambda_{\mathrm{g}}$ ) because our of-the-self T-Junctions have arms longer than $0.042 \lambda_{\mathrm{g}}$ and thus, we add half a guided wavelength. Despite that $r=0.542 \lambda_{\mathrm{g}}$ is not the optimal one (point B), it provides adequately low reflection coefficient, i.e. lower than $-40 \mathrm{~dB}$, as it can be deducted (point C) from Fig. 8 .

\section{B. Optimization of the FMPC dimensions}

Our goal is to design, integrate and experimentally verify an FMPC that utilizes the above designed waveguide turn $\left(w=0.310 \lambda_{\mathrm{g}}, r=0.542 \lambda_{\mathrm{g}}\right)$ and has main and secondary cavity lengths approximately equal to $L \approx 2.0 \lambda_{\mathrm{g}}$. In order to use already available of-the-shelf waveguide components for the integration, we define a secondary cavity length $L_{2}=2.076 \lambda_{\mathrm{g}}$, whereas the main cavity length $L_{1}$ is properly optimized by using the equivalent-circuit model in order to achieve the maximum possible cavity-gain.

Fig. 9 presents the equivalent-circuit calculations for the main cavity length $L_{1}$, the input iris reactance $X$ (normalized to $\mathrm{Z}_{0}$ ), the main cavity gain $G$ and the output power leakage $L_{\text {out }}$ with respect to the short-circuit length $r_{\text {out }}$ of the output Tjunction (Fig. 4) that controls the leakage. According to Fig. 9, by selecting the leakage short-circuit length $r_{\text {out }}=0.357 \lambda_{\mathrm{g}}$, iris opening $w_{\text {iris }}=0.190 a$ (where $a$ is the broad side of the waveguide) and main-cavity length $L_{1}=2.352 \lambda_{\mathrm{g}}$, the leakage is $-40 \mathrm{~dB}$ and the corresponding cavity gain is $G=26 \mathrm{~dB}$. For the same $w_{\text {iris }}$ and $r_{\text {out }}$ values, full-wave calculations with CST Studio Suite (Fig. 10) indicate an optimal main cavity length equal to $L_{1, \mathrm{CST}}=2.354 \lambda_{\mathrm{g}}$, which corresponds to relative error

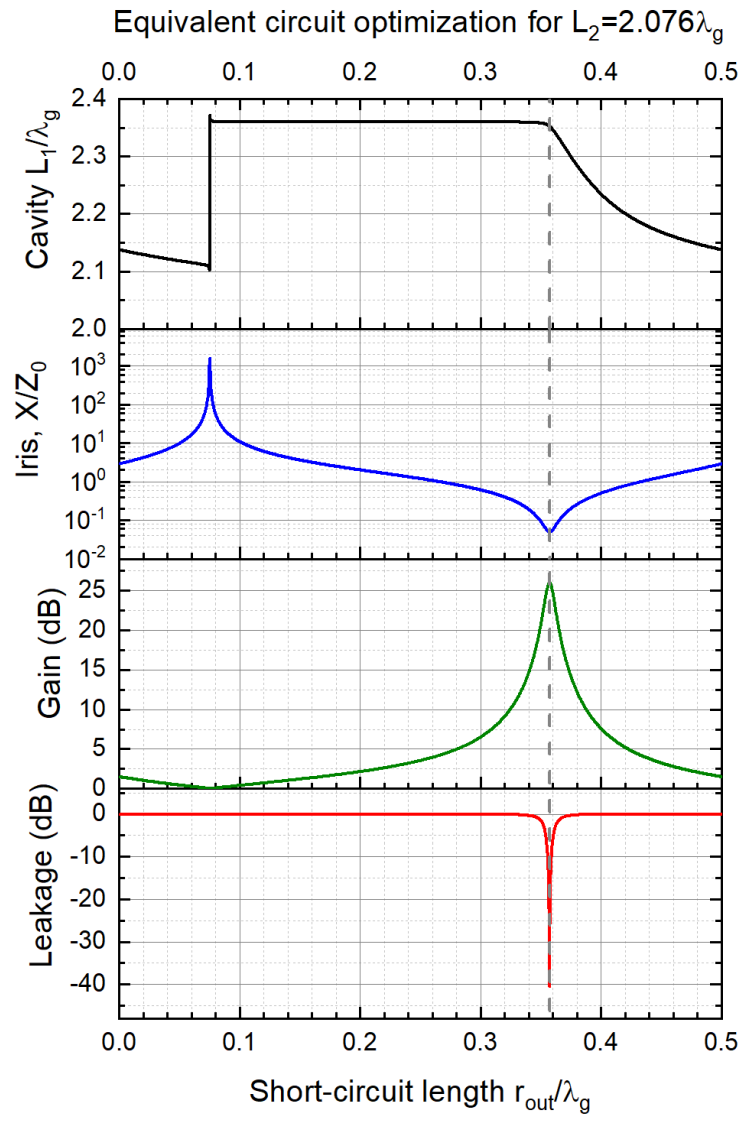

Fig. 9. Main cavity length $L_{1}$, iris reactance $X / Z_{0}$, main cavity gain $G$ and compressor leakage $L_{\text {out }}$ with respect to the output short-circuit length $\mathrm{r}_{\text {out }} / \lambda_{\mathrm{g}}$.

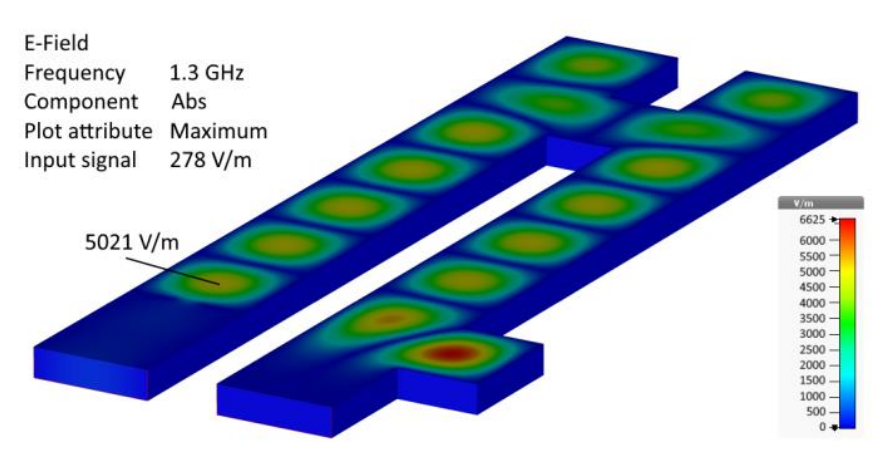

Fig. 10. Full wave simulation of the electric field magnitude $|\mathrm{E}|$ for the case of the optimal design of the H-plane waveguide turn. The amplitude of the first maximum close to the iris is $5021 \mathrm{~V} / \mathrm{m}$ and corresponds to gain $25.1 \mathrm{~dB}$, for excitation signal with amplitude $278 \mathrm{~V} / \mathrm{m}$.

$0.08 \%$. Considering that in the full-wave simulation the standing wave magnitude has amplitude $5021 \mathrm{~V} / \mathrm{m}$ and the excitations signal has amplitude $278 \mathrm{~V} / \mathrm{m}$, the CST calculated gain is $G_{\text {CST }}=25.1 \mathrm{~dB}$. The full-wave calculated gain is in good agreement with the equivalent circuit, considering that the later does not take into account higher-order phenomena and losses for the T-junction's core part. The simplification regarding the losses is the main reason for the above discussed gain overestimation. Indeed, by neglecting the conductive losses of the T-junction center part, the full-wave simulation gives gain $G^{\prime}{ }_{\text {CST }}=25.9 \mathrm{~dB}$, which is essentially identical with the circuit model estimation. 


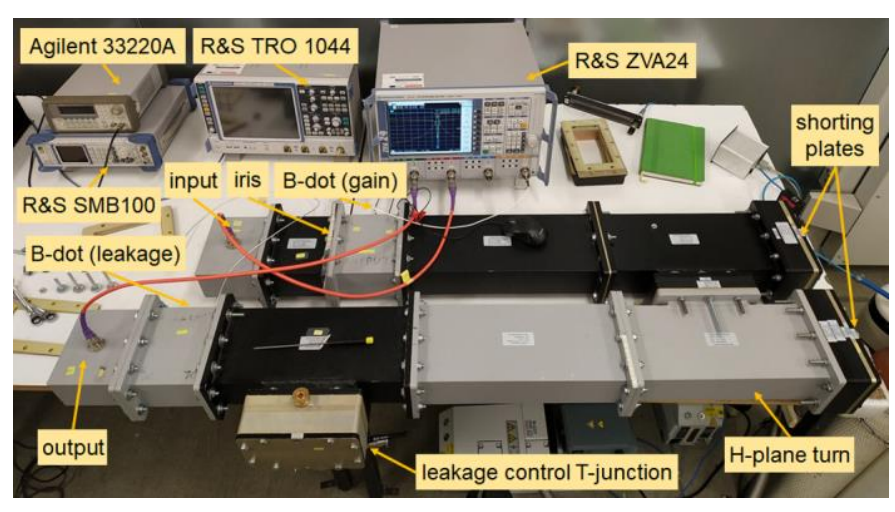

Fig. 11. Experimental setup for the verification of the design with the Vector Network Analyzer. The dimensions of the FMPC are $w_{\text {iris }}=33.0 \mathrm{~mm}$, $\mathrm{L}_{1}=753.7 \mathrm{~mm}, \mathrm{r}_{\text {turn }}=174.5 \mathrm{~mm}, \mathrm{w}_{\text {turn }}=99.8 \mathrm{~mm}, \mathrm{~L}_{2}=669.0 \mathrm{~mm}$.

It should be noted that the total calculation time for Fig. 9, which includes at least $5000(r, L, X)$ triplets, is less than $1 \mathrm{~s}$ (on an Intel i5 laptop computer), whereas for a single triplet the 3D full-wave calculation takes at least $1 \mathrm{~min}$, even when symmetries are taken into account in order to reduce the mesh size. In addition, due to the very high quality-factor that the FMPC has, it is at least cumbersome to find the optimal triplet $\left(r_{\text {out }}, w_{\text {iris }}, L_{1}\right)$ without having a relatively accurate initial guess, as the one that the equivalent circuit provides.

\section{EXPERIMENTAL VERIFICATION OF THE $1.3 \mathrm{GHZ}$ FMPC}

Fig. 11 presents the laboratory test-bench that is used for the experimental verification of the proposed $1.3 \mathrm{GHz}$ FMPC design. The dimensions of the depicted waveguide components are $w_{\text {iris }}=33 \mathrm{~mm}(0.2 a), L_{1}=753.7 \mathrm{~mm}\left(2.339 \lambda_{\mathrm{g}}\right)$, $r=174.5 \mathrm{~mm}\left(0.554 \lambda_{\mathrm{g}}\right), w=99.8 \mathrm{~mm}\left(0.310 \lambda_{\mathrm{g}}\right), L_{2}=669 \mathrm{~mm}$ $\left(2.076 \lambda_{\mathrm{g}}\right)$ and $r_{\text {out }}=115 \mathrm{~mm}\left(0.357 \lambda_{\mathrm{g}}\right)$. Almost all the dimensions of the experimental components are practically identical with the estimated ones by the circuit model and the full-wave calculations. The small deviation of the iris opening, which is slightly wider, and the length $L_{1}$, which is slightly shorter, could be addressed to the relatively loose connection of the iris with the rest of the waveguide components. In particular, the iris consists of two independent sliding metallic plates, a choice that simplifies and accelerates the adjustment of the iris' opening dimensions. However, such a construction does not allow to use all the screws on the waveguide flange, is prone to misalignments of the iris' edges and does not ensure a perfectly symmetric iris opening, which in turn make the equivalent-circuit model as well as the 3D simulation of the iris less accurate. This is reflected in the experiment on the main cavity length $L_{1}$, since this is the last component to be tuned in order to ensure a strong resonance.

The auxiliary equipment and the different configurations that are used for the measurements are described in detail in [18], where the performance of a SMPC system is optimized. For the sake of brevity, herein, we present briefly the experimental topology and the measurement procedure in order to focus on the achieved performance. As depicted in Fig. 11, the FPMC cavity is implemented between the iris and the leakage control T-junction. Measurements are taken with the use of two B-dot sensors: one placed at a distance of $\lambda_{g} / 4$

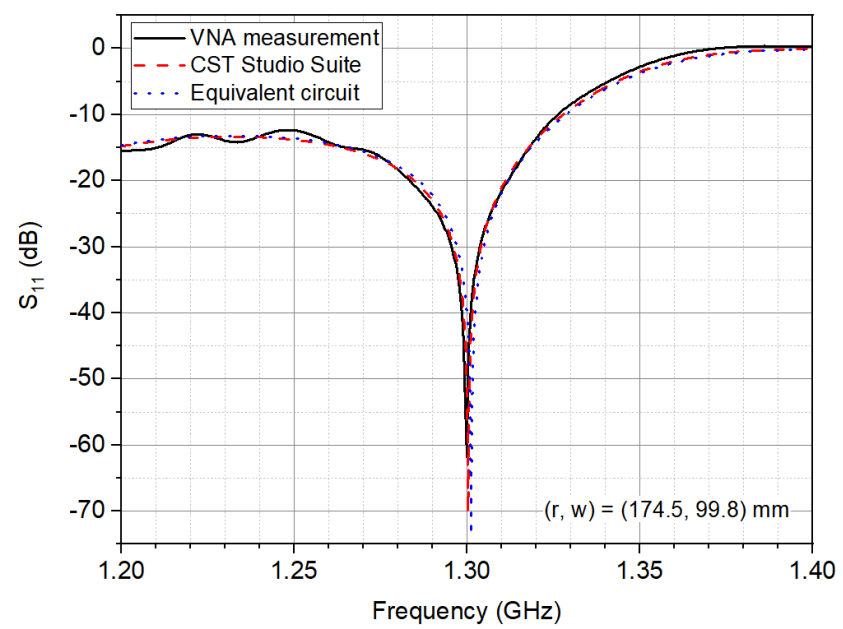

Fig. 12. Reflection coefficient $S_{11}$ of the WR650 H-plane waveguide turn that is used for the experimental verification of the developed FMPC system.

from the iris in order to measure the standing wave maximum and thus the cavity gain and the other after the leakage control $\mathrm{T}$-junction in order to measure the leakage during cavity charging or the output pulse during the cavity discharging. Low-power measurements are performed with a 4 port $24 \mathrm{GHz}$ Vector-Network-Analyzer (VNA). VNA Port 1 is connected to the coaxial to waveguide converter, whereas port 3 and 4 to the two B-dot sensors (similarly to Fig. 2 in [18]). High-power measurements are performed using a TravellingWave-Tube Amplifier (TWTA) with a nominal output of 1 $\mathrm{kW}$ and a 4 port $4 \mathrm{GHz}$ oscilloscope (similarly to Fig. 6 in [18]). Using the 4 ports of the oscilloscope we monitor the cavity standing-wave and the leakage/output-pulse through the two B-dot sensors, while the input and reflected signals are monitored with the use of a directional coupler connected at the coaxial-to-waveguide adapter. The statistics function of the oscilloscope is used to monitor the variations of the cavity standing wave as well as of the leakage/output pulse level by making subsequent discharges of the cavity.

Before assembling the complete FMPC system, the H-plane waveguide-turn design is verified by VNA measurements. The VNA is calibrated properly in order to account for the coaxialto-waveguide fixtures. As shown in Fig. 12, the measured reflection coefficient $S_{11}$ is in very good agreement both with the equivalent-circuit calculations and the 3D full-wave simulation. The minimum of the circuit-based results appears roughly $1 \mathrm{MHz}$ higher, since, as it was mentioned in Section II, the used $r$-value in the experiment is slightly smaller than the optimal one.

Fig. 13 presents VNA measurements for the input reflection coefficient $|\rho|$, the leakage $L_{\text {out }}$ and the cavity gain $G$ with respect to the frequency. In this configuration, we assess the performance of the FMPC just in cavity-charging conditions, i.e. the plasma switch has atmospheric pressure and it is not activated. At $1.3 \mathrm{GHz}$ resonance, we measure $|\rho|_{\mathrm{VNA}}=-24 \mathrm{~dB}$, $L_{\mathrm{VNA}}=-35.6 \mathrm{~dB}$ and $G_{\mathrm{VNA}}=24 \mathrm{~dB}$. Considering the number of waveguide spacers that we use and the small differences of the as-built to the as-designed dimensions, the experimentally measured cavity gain is in very good agreement with the 


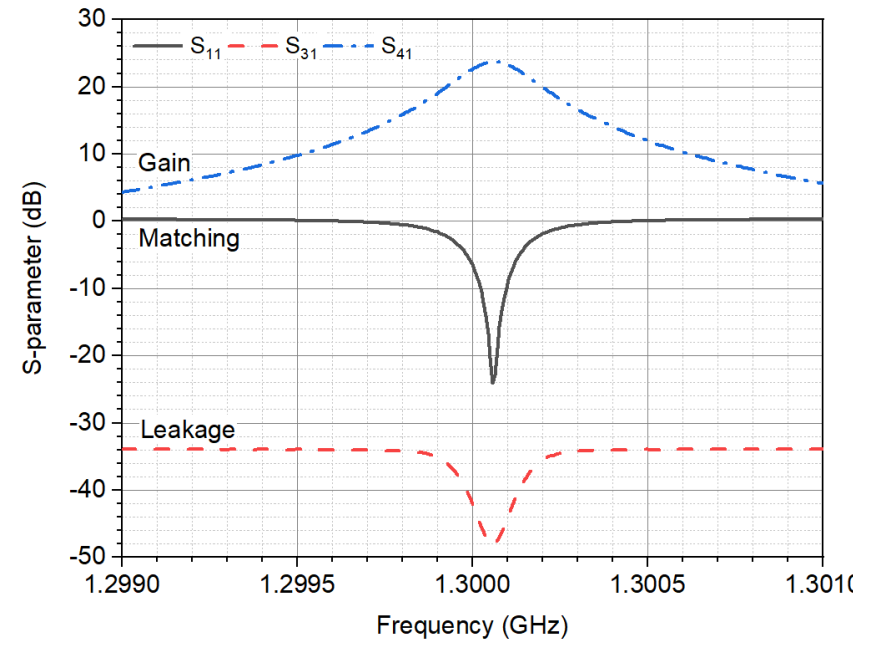

Fig. 13. VNA measurements for the matching $|\rho|$, the leakage $L_{\text {out }}$ and the gain $G$ of the designed FMPC cavity. At $1.300 \mathrm{GHz}$ we achieve $|\rho|_{\mathrm{VNA}}=-$ $24 \mathrm{~dB}, L_{\mathrm{VNA}}=-35.6 \mathrm{~dB}$ and $G_{\mathrm{VNA}}=24 \mathrm{~dB}$.

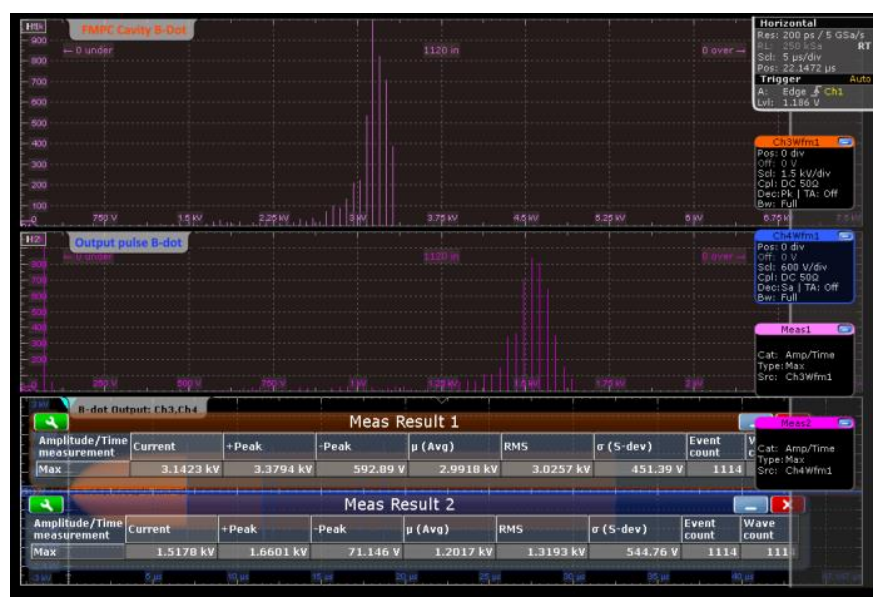

Fig. 14. Oscilloscope screenshot for the evaluation of the charging and compression performance (statistics representation).

design, especially when the losses of the core part of the Tjunctions are considered in the modeling $\left(G_{\mathrm{CST}}=25.1 \mathrm{~dB}\right)$.

The measurement of the extraction gain is a more complex procedure and depends strongly on the design and operational parameters of the plasma switch [18], i.e. on the geometry of the gas tube, as well as on the gas type, flow and pressure. A detailed study on the optimization of the extraction performance of the FMPC is considered to be out of the scope of this work. Thus, in order to assess the extraction gain of the developed system and confirm the capability to create longer pulses with a small form-factor folded cavity, we follow the guidelines proposed in [18], where a SMPC system is optimized in terms of the output-pulse power and the pulse repetition frequency (PRF). In particular, we use a Teflon made plasma-switch with diameter $10 \mathrm{~mm}$ filled with natural air, a top electrode with transverse gas flow, gas pressure 1 Torr and flowrate $120 \mathrm{sccm}$. Fig. 14 presents the statistical analysis of multiple cavity charging/discharging events that are detected by the oscilloscope. The PRF is set to $150 \mathrm{~Hz}$. According to the B-dot measurements, the standing wave in the cavity has average amplitude $V_{\text {cav }}^{\text {avg }}=2992 \mathrm{~V}$ and peak

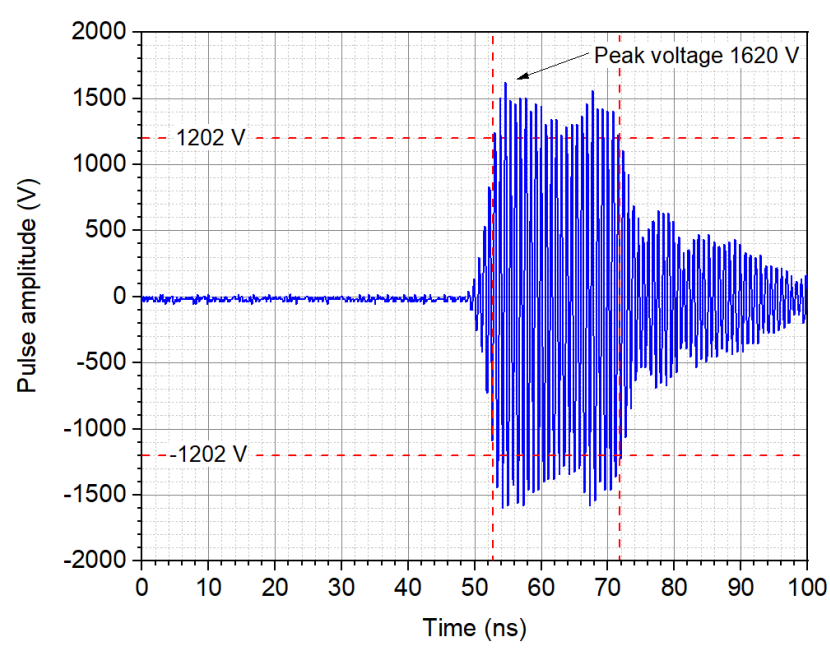

Fig. 15. Oscilloscope recording of an output pulse with duration approximately $21 \mathrm{~ns}$ and amplitude $1200 \mathrm{~V}$.

amplitude $V_{c a v}^{\max }=3379 \mathrm{~V}$. The compressed pulse has average amplitude $V_{\text {out }}^{\text {avg }}=1202 \mathrm{~V}$ and peak amplitude $V_{\text {out }}^{\max }=1660 \mathrm{~V}$. The FMPC system is driven by a $1 \mathrm{~kW}$ TWTA. Considering that the input voltage is $V_{\text {in }}^{\text {avg }}=223 \mathrm{~V}$, the cavity gain during the charging is on the average $G_{c a v}^{\text {avg }}=22.6 \mathrm{~dB}$ and peaks out at $G_{\text {cav }}^{\max }=23.6 \mathrm{~dB}$. Note that despite that the GDT properties were not carefully optimized, the maximum gain is essentially the one expected by the VNA measurements $\left(G_{\mathrm{VNA}}=24 \mathrm{~dB}\right)$. Similarly, the extraction gain is on the average $G_{\text {out }}^{\text {avg }}=14.6 \mathrm{~dB}$ and peaks at $G_{\text {out }}^{\max }=17.4 \mathrm{~dB}$. Under ideal switching conditions the extraction gain differs from the cavity gain by $6 \mathrm{~dB}$. In our experiments, the difference between the maximum and the average cavity and extraction gain is $6.2 \mathrm{~dB}$ and $8 \mathrm{~dB}$, respectively. The variation of the so called extraction loss in the range of $0.2 \mathrm{~dB}-2 \mathrm{~dB}$ coincides with the typical performance of a straight MPC system [12]. In this sense, this is an indirect confirmation that, when properly designed, the folding of the cavity does not deteriorate the MPC performance.

Fig. 15 presents a pulse with amplitude (during the flattop) at least equal to the average one, i.e. higher than $1202 \mathrm{~V}$. The measured pulse duration is approximately $21 \mathrm{~ns}$. Note that the total waveguide length (see Fig. 10) that forms the cavity is roughly $L_{\text {total }}=\left(L_{1}+L_{2}+w+2 \cdot r\right) \cdot \lambda_{\mathrm{g}}+2.5 \cdot a=2284 \mathrm{~mm}$. The last part of the summation, i.e. 2.5.a, corresponds to the contribution of the three T-junctions that are used. Note that the T-junction that controls the leakage of the compressor has a minimum approximately in the middle of the side arm [14], thus the cavity border on the output side is considered to be the center of the T-junction. This total length corresponds roughly to an output pulse duration $\tau_{\text {out }}=2 \cdot L_{\text {total }} / v_{\mathrm{g}} \approx 21 \mathrm{~ns}$, where $v_{\mathrm{g}}=2.146 \cdot 10^{8} \mathrm{~m} / \mathrm{s}$ is the group velocity of the guided wave. In order to achieve a similar pulse duration with an SMPC we would need a main cavity with physical length $\mathrm{L}_{\mathrm{SMPC}} \approx 7.209 \cdot \lambda_{\mathrm{g}}+0.5 \cdot \mathrm{a}=2405 \mathrm{~mm}$, according to equivalent circuit calculations [9]. The estimated SMPC gain is 
$\mathrm{G}_{\mathrm{SMPC}}=25 \mathrm{~dB}$, which is almost the same with the one $\left(G_{F M P C}=26 \mathrm{~dB}\right)$ of the FMPC under study. The $1 \mathrm{~dB}$ difference can be addressed to the more accurate losses representation of the SMPC model, since fewer T-junctions are involved. For the tested FMPC, the longer physical dimension is the secondary cavity side, which has a total length equal to $L_{\max }=\left(L_{2}+r\right) \cdot \lambda_{\mathrm{g}}+1.5 \cdot \mathrm{a}=1091 \mathrm{~mm}$. It is evident that the performance of the FMPC is equivalent to the SMPC one, while the maximum length of the compressor has been reduced by a factor of two.

\section{CONCLUSION}

An equivalent-circuit/transmission-line model was used for the design of a microwave pulse compressor that uses waveguide-turns in order to reduce the maximum (length) dimension of the resonant cavity. Although the mathematical formulation presented refers to $\mathrm{H}$-plane turns made by combining two T-junctions, the approach is universal and can be easily modified to address any number and type of waveguide components that have a proper equivalent circuit. It is important to note that by carefully designing the turns, a broadband behavior can be achieved making the compressor less insensitive to the manufacturing tolerances.

As an example, an H-plane FMPC using a waveguide turn made of T-Junctions was designed and experimentally tested. Full-wave simulations and experimental measurements confirm that the circuit-based calculations are accurate and fast. The achieved performance of the FMPC is similar to the one of an SMPC while its physical length is two times shorter.

\section{REFERENCES}

[1] C. Nantista, Z. D. Farkas, N. M. Kroll et al., "High power RF compression with SLED-II at SLAC," Particle Accelerator Conference, 487 Washington, DC, May 17-20, 1993. SLAC-PUB-6145, Apr. 1993

[2] M. K. Thumm and W. Kasparek, "Passive high-power microwave components," IEEE Trans. Plasma Sci., vol. 30, no. 3, pp. 755-786, Jun. 2002 .

[3] J. Benford, J. A. Swegle, and E. Schamiloglou, High Power Microwaves, 2nd ed. New York, NY, USA: Taylor \& Francis, 2007

[4] G. Burt, S. V. Samsonov, A. D. R. Phelps et al., "Microwave Pulse Compression Using a Helically Corrugated Waveguide," IEEE Trans. Plasma Sci., vol. 33, no. 2, pp. 661-667, Apr. 2005.

[5] E. G. Farr, L. H. Bowen, C. E. Baum, and W. D. Prathe, "Microwave Pulse Compression Experiments at Low Power", in Proc. URSI Gen. Assembly, 2008, pp.459-462.

[6] L. Beilin, A. Shlapakovski, and Ya. E. Krasik, "Resonant microwave pulse compressor operating in two frequencies," J. Appl. Phys., vol. 114, no. 034503, pp. 1-8, Jul. 2013.

[7] R. A. Alvarez and D. P. Byrne, "Prepulse Suppression in Microwave Pulse-Compression Cavities," Rev. Sci. Instrum, vol. 57, no. 10, pp. 2475-2480, Oct. 1986.

[8] E. G. Farr, L. H. Bowen, W. D. Prather, and C. E. Baum, "Microwave pulse compression experiments at a low and high Power," Circuit and Electromagnetic System Design Notes-Note 63, Jan. 2010.

[9] S. P. Savaidis, Z. C. Ioannidis, S. A. Mitilineos and N. A. Stathopoulos, "Design of Waveguide Microwave Pulse Compressors Using Equivalent Circuits", IEEE Trans. Microw. Theory Tech., vol. 63, no. 1, pp. 125134, Jan. 2015.

[10] S. N. Artemenko, S. A. Gorev, V. S. Igumnov, and R. E. Beverly, "High-power microwave pulse compressors with a variable geometry of accumulative resonant cavity," in Proc. Int. Conf. Pow. Modul. High Volt., Jul. 6-9, 2016, pp. 275-278.

[11] S. N. Artemenko, S. A. Gorev, V. S. Igumnov, and G. Y. Yushkov, "Formation of long nanosecond rectangular pulses in the active RF pulse compression system with a compact resonant cavity," J. Phys.: Conf. Ser., vol. 830, no. 012030, pp. 1-9, 2017.

[12] S. N. Artemenko, V. S. Igumnov, and R. E. Beverly, III, "The study of compact microwave pulse compression systems", in Proc. Pacific Symp. Pulsed Power Appl., Waikoloa Beach, Hawaii, pp. 12, Aug. 2017.

[13] S. N. Artemenko, V. S. Igumnov, A. S. Shlapakovsky and Y. G. Yushkov, "Compact Active S-Band Microwave Compressors for Producing Rectangular Pulses of Up To 100 ns", IEEE Trans. Microw. Theory Tech., vol. 67, no. 2, pp. 597-605, Jan. 2019.

[14] S. P. Savaidis, Z. C. Ioannidis, N. A. Stathopoulos, S. A. Mitilineos and C. Tsitouri, "Transmission line modeling of active microwave pulse compression systems," Int. Conf. Microw., Commun., Antennas Electron. Syst., Oct. 21-23, 2013, pp. 1-5.

[15] Z. C. Ioannidis, S. P. Savaidis, S. A. Mitilineos, C. Tsitouri and N. A. Stathopoulos, "Equivalent circuit/transmission line model of microwave pulse-compression cavities," Int. Symp. Electromagn. Compat., Sep. 1-4, 2014, pp. 134-139.

[16] N. Marcuvitz, Waveguide Handbook, IEEE Electromagnetic Waves Series, Peter Peregrinus Ltd, 1986.

[17] D. M. Pozar, "Microwave Engineering", $2^{\text {nd }}$ Edition, New York, Wiley, 1988.

[18] S. Savaidis, S. Mitilineos, Z. C. Ioannidis, and N. Stathopoulos, "Experiments on the Pulse Repetition Frequency Optimization of a $1.3 \mathrm{GHz}, 100 \mathrm{~kW}$ Microwave Pulse Compressor", IEEE Trans. Microw. Theory Tech., early access online, March 2020.

Z. C. Ioannidis received the BSc. in physics, in 2002, the MSc. in electronics and radioelectrology, in 2004, and the Ph.D. in 2008, from the National and Kapodistrian University of Athens, Greece. Since 2016 he is with the Institute for Pulsed Power and Microwave Technology at the Karlsruhe Institute of Technology, Germany.

S. P. Savaidis received the E.E. Diploma and Ph.D. degrees from the National Technical University of Athens, Greece, in 1991 and 1997, respectively. He is a Professor with the Department of Electrical \& Electronics Engineering, University of West Attica, Greece.

S. A. Mitilineos received the Diploma in Electrical \& Computer Engineering and his $\mathrm{PhD}$ from the National Technical University of Athens, Greece, in 2001 and 2007, respectively. He is currently an assistant professor with the Department of Electrical \& Electronics Engineering, University of West Attica, Greece.

S. Livieratos received the E.E. Diploma and the Ph.D. degree from the National Technical University of Athens, Greece, in 1992 and 1998, respectively. He joined the School of Pedagogical and Technological Education, Department of Electrical \& Electronic Engineering, Greece, in 2009, where he has been a Professor since 2018 .

N. A. Stathopoulos received the E.E. Diploma and the Ph.D. degree from the National Technical University of Athens, Greece, in 1984 and 1995, respectively. Since 2008 he has been a Professor with the Department of Electrical \& Electronics Engineering, University of West Attica, Greece. 